alcohol, instead of a dilution. The patient could only tolerate three minims at first on account of the pain, but later endured with difficulty 30 minims. Dr. Kuh thinks the diluted form might have been better. The tumor was a carcinoma seated in the naso-pharynx. The reduction in size began after the seventh injection, and was apparently cured after the twenty-fifth. After four months there was no sign of return. He put the fluid into the tumor itself.

I have recommended the alcohol injections in consultation in several cases; but there has not been time enough yet to judge of the permanency of the cure, nor of the effects on the infected lymphatic glands. At present I advise it only in inoperable cases.

Dr. Young of Bloomfield, Iowa, uses the pure alcohol and puts it into all parts of the tumor itself. $\mathrm{He}$ claims some cures.

Hué of France, injects a solution of arsenic and cocain at intervals of two or three days, and later at longer periods. He claims good results and some cures, with freedom from pain.

Alexandro and others have injected methyl blue, and report the pains relieved and the tumors made to shrink and disappear.

Dr. C. M. Fenn of California, reports the injection of saturated solution of citric acid. He says it brings "instant relief" and some cures.

This whole investigation is yet in a crude and unfinished state, but it will go on to more definite results. If some injection can be found which, while curing the primary tumor, will have such a controlling power over the inaccessible secondary ones, as is claimed for the chelidonium, it will be of immense value. If this power over the infected glands is not finally proven, it will still be of great importance in many individual cases to atrophy the primary tumor and to relieve the distressing pains.

3912 Lake Avenue.

\section{SURGICAL SHOCK.}

Read before the Chicago Academy of Medicine April 9, 1897. BY FENTON B. TURCK, M.D. CHICAGO.

The experiments on animals for inducing shock were reported before the American MEdical AssoCIATION, May, 1896. In these experiments the color changes in the abdominal viscera were noted, the blood pressure in both arteries and veins in the peripheral circulation as well as the central. I will give a brief résumé of some of these experiments.

Experiment No. 1 showed that after exposure of the stomach and intestines to draught of air from an open window, temperature 58, profound shock resulted. The gastric veins within half an hour were all congested. Respiration shallow; pulsations weak. The chloroform was pushed to the extreme; animals stopped breathing. Withdrawal of chloroform and artificial respiration did not excite respiratory movements. The intragastric resuscitator ${ }^{1}$ was introduced into the stomach, and hot water $130 \mathrm{~F}$. As soon as the water returned from the other side of the double tube, and more hot water introduced the animal began to breathe. In twenty minutes there was a remarkable change in the color of the stomach. Instead of the blue dull color it became a bright red. In forty minutes the surface of the body became warm. The mouth showed a bright red color, in place of the pale

\footnotetext{
1 For description of the instrument see Journal, Jan. 11, 1896, p. 79.
}

livid hue. Pulsation became strong, showing increased arterial tension and the respirations became deep and uniform. This was repeated during the same period, and the second time the same phenomena were again presented. When the restoration of the circulation seemed assured, the abdominal wound was closed, leaving a permanent gastric fistula. The animal recovered and was exhibited in my lectures given at Jefferson Medical College, Philadelphia, February, 1896.

Experiment No. 2 was of a similar character. The pressure in the veins and the arteries was studied with a manometer to indicate the changes in blood pressure. During shock the veins and the viscera show increased pressure while the arteries a decrease. After the introduction of the intragastric resuscitator this condition is reversed.

Experiments 3 and 4 similar to 1 and 2 .

Experiment 5 was a study of changes that occur by the observation of color of the viscera. The shades from the normal to profound shock, showing eight different stages; also standard changes of shades observed after the introduction of the intragastric resuscitator. Referring to the chart, No. 1 is the normal, No. 2 the first changes observed after shock, No: 3 and so forth up to 8 . It is found that within three minutes after exposure shade No. 2 was observed, in eight minutes No. 3; ten minutes No. 4 ; fifteen minutes No. 5; half an hour No. 6. It required an hour and a half for No. 8 to appear. There was a marked difference in the shades of the intestine and the stomach; when the stomach showed shade No. 5 the intestines appeared shade No. 6 (after the introduction of the intragastric resuscitator). After thirty minutes the shade in the stomach showed No. 4, intestines showed No. 5 . In forty-five minutes all the larger veins were apparently empty. In one hour the stomach showed shade No. 3, while intestines remained shade No. 4. (Exhibition of color chart.)

SHOCK BY THE INTRODUCTION OF TOXINS.

Experiment No. 5. Method. Stomach contents drawn off, from a patient suffering with dilatation of the stomach. One hundred cu. cm. injected into a dog weighing $15 \mathrm{~kg}$. This is repeated in one hour. Evidence of shock observed. In one hour the surface was cold, the mouth and gums pale, and on opening the abdomen a deep injection of all the viscera was apparent. The animal was in shock. Introduction of the intragastric resuscitator, reduction of the shock is produced. The animal recovered. While a control under the circumstances carried out on a dog weighing $16 \frac{1}{\mathrm{~kg}}$. the animal died.

Experiment No. 7. Shock produced by operation on abdominal viscera. Long incision from xiphoid appendix to symphysis pubis. Operation for gastrostomy (Turck's valve operation). Operation for the Heincke-Mukilitz pyloplastic. Also Martell's operation for gastro-enterostomy. Within one hour the entire stomach and entestines showed a bluish color with all the veins congested. Respiration shallow; pulse 130; evidence of collapse. Hot water bag placed inside the stomach. Temperature of 125 raised to 135 , in a moment there was a change in both the pulse and respiration, the high heat having an instantaneous reflex action. The color changes within two minutes. In fifteen minutes the veins grew smaller in size and the surface appeared more of a rosy color. Respirations not so shallow, pulse strong, show- 
ing increased tension. Twenty minutes intestines death and anesthesia of the surface, pale and livid. began to change in color similar to the effect observed The intragastric resuscitator was introduced into the in the circulation of the stomach. The general effect stomach; within ten minutes the pulse became fuller, was pronounced upon all the mesenteric vessels. stronger and less rapid. The color began to change Thirty minutes the congestion was reduced and arte- in the lips from a bluish to a reddish. Within half rial circulation restored. Water introduced had to be an hour the surface became rosy in color, hands and changed every five minutes and absorption of heat feet warm, and instead of the heat increasing perspiwas very great. Water introduced at 125 and 130 returned at 110 and 105 . The viscera was exposed for three hours. The animal made a good recovery.

Experiment No. 9. To determine the rapidity with which color changes take place in the abdominal viscera. Exposed to the cold draught No. 1 on opening the abdomen. Color No. 2 in five minutes; No. 3 in ten minutes; 4 in fifteen minutes; 5 in thirty minutes; 6 in forty-five minutes. Hot water bag introduced in ten minutes from the time shade No. 2 was evident. In other areas No. 3 shade was observed.

Experiment No. 10, April 7, 1896; assisted by Captain Appell, Assistant Surgeon U. S. A. The animal was placed on a hot water bed. The abdomen was opened to a draught of air; operation was made for valvular gastrostomy. In forty-five minutes profound congestion of abdominal viscera was apparent; congestion of all the viscera, gastric vessels, gastric epiploica, and senestra, and gradually became filled and the tortuous veins stood out in dark relief upon the livid background. The stomach, though congested, was cold to the touch and intestines likewise. All evidence of shock was present. Reflexes absent though the animal was not under the influence of chloroform at this time, or ether. Shallow and rapid breathing. Rapid weak pulse, surface of the body cold, gums and tongue pale; collapse was imminent. The intragastric resuscitator was introduced and water of 130 degrees. In three minutes the water was withdrawn at 110 degrees $F$. The effect of the hot water was instantaneous. A continuous stream was allowed to play through for ten minutes; breathing and pulse improved at once and the circulatory changes were manifest, probably due to the stimulation of the solar plexus, cardiac plexus. The veins diminished in size right under the eye, the red bluish shade taking place instead of the sluggish blue. In twenty minutes the animal breathed steadily, pulse "came up," and arterial tension was increased. The body grew warm; tongue and gums showed a natural color. It was evident to all present that the animal was out of shock in half an hour.

REPORT OF CASES AND METHOD OF TREATMENT.

The following cases are selected from a large number to illustrate the symptoms and physical condition found in shock, also the effects of the treatment by the intragastric resuscitator.

Case I was that of a woman 40 years of age, suffering from impaction in which she was found in an unconscious condition, surface of the body cold, pale, lips bluish. The intragastric resuscitator was immediately introduced into the stomach. Within fifteen minutes reaction was complete, consciousness was restored and in thirty minutes the surface of the body, hands and feet were warm and the patient out of shock. Following this the usual treatment was inaugurated to reduce the impaction of the bowel.

Case 2.-Mrs. K., age 36, was found in shock, resulting from acute gastro-enteritis. There was a cold sweat upon the surface, a feeling of impending

Can, skin became more dry while under shock.

Case 3, operation on a case of carcinoma of the pylorus. The operation was a gastro-enterotomy. Murphy button anastomosis. On opening the abdomen on exposure to the air a red shade of the stomach was observed, and after manipulation of the viscera there were great adhesions, shade No. 3 was observed. In thirty minutes shade No. 4 was observed. At the same time breathing became vary laborious, pulse diminished, the lips became pale and there were other evidences of shock. The intragastric resuscitator was introduced into the stomach and the imme. diate effects could be observed under the eye. The change of the color of the viscera was apparent; with the reduction of the venous congestion, surface grew warm, the perspiration which stood out like beads upon the forehead, disappeared. All signs showed the reduc. tion of shock within fifteen minutes.

Case 4.-A young football player in Lincoln Park was struck in the epigastrium. At the same time sustained a Colle's fracture. Was brought into the office insensible and after a noment recovered, call. ing for water; his eyes were leaden; slow respiration; surface cold; great anxiety. I introduced the intragastric resuscitator and the effect was immediate. The breathing became even, the pulse that was weak became strong, fuller, the color returned to the surface; the eyes bright. The effect of this was very evidently due to the use of the intragastric resuscitator.

Case 5.-Mrs. MoG., age 45, case hemorrhage from the bowels; estimated loss of blood was over one quart. All the signs of shock were present including anesthesia, disturbance of vision, thirst, cold surface, lips blue, anxious expression; patient experiences the feeling of impending death. The intragastric resuscitator was introduced into the stomach and within twenty minutes the symptoms disappeared with the exception that the hands and feet were still cold, but within forty-five minutes the hands and feet also were warm. No other treatment for the reduction of shock was used. This was again repeated the next day and the patient felt so well after treatment that she requested a daily treatment, which was instituted for one week. Recovery complete.

Case 6.-Mrs. F., Presbyterian Hospital, called by Dr. Etheridge; been in shock two days and a half. The patient was moribund, mottled blue surface, could not speak, and impending death was apparent. The intragastric resuscitator was introduced in the usual manner and temperature at 130; observations made by the attending and house physician as well as myself. It was observed that the stimulation was immediate. The pulse that was before imperceptible could now be felt. Breathing improved and patient could speak. The physicians and attendants thought that there might be some hope for her recovery, but as the patient was already moribund, the blood having passed largely into the veins, and extravasation had occurred and the whole surface was mottled. When in this condition it seemed that nothing could restore her. The improvement in this case kept up for three 
hours, when the patient gradually sank and died. It was instructive in this case, however, to observe in the extreme the profound effect that the hot water had upon the nervous centers and upon the circulation, including respiration and return of intelligence.

The seventh, eighth and ninth cases were cases of laparotomy upon the pelvic viscera. Shock was imminent in each case and in Case 9 the shock occurred also the following day, when the intragastric resuscitator was introduced again with the usual prompt results.

The last case that I report here tonight is one for an exploratory incision in which was found large adhesions, due to a pancreatitis, parapancreatitis hemorrhagica. The case was operated upon by Dr. Ferguson. The patient was found in shock; face was blue, especially marked about the lips; breathing became very labored, large beads of perspiration were found all over the face and was regarded as a case of shock. The intragastric resuscitator was introduced into the stomach and immediately the effect was manifest to all present. Within five minutes the color had changed from the bluish to the rosy tint. The perspiration disappeared from the face, breathing was again deep and regular, pulse restored. It was evident that the respiration of this condition was brought about promptly by the use of the intragastric resuscitator.

$$
\text { DISCUSSION. }
$$

Dr. James G. KiERnan-I can hardly agree with Dr. Turck that stimulation of the ano genital center is comparable with that of the gastric centers. It has been shown by experiments on conditions of collapse resulting after anesthesia, and also on the conditions of coma resultant on opium poisoning, that the ano-genital center is one of the last centers in the body whose reflex action is destroyed, and is the quickest to react to excitants in the restoration of consciousness. For example, sixteen years ago I was called to a case of opium poisoning. The patient, a prohibition Congressman, went on a tremendous spree, as a consequence of which he had incipient symptoms of delirium tremens, and sent out for laudanum and took an ounce of it, All the usual methods to counteract poisoning from opium were employed without avail. The case was turned over to me with the benevolent intention of having me sign the death certificate, inasmuch as he had passed into the condition of opium collapse. I poured three drams of tincture of capsicum up his rectum. I have never seen anything act as quickly as that did. Since that case a large number of others have been reported independently, and my observations have confirmed with regard to the quickness with which this center reacts. Not only that, but a large number of observers have pointed out that this center is the quicker to be excited in failures after anesthesia than almost any other. This has been demonstrably the case with Dr. R. A. Murray in New York and a number of others who have followed his researches. I do not doubt, from the surrounding innervation of the stomach, but what Dr. Turck would get undeniably rapid and beneficial effects from this procedure. At the same time, I doubt very much whether if the shock had passed beyond a certain point, he would get quite as rapid and efficacious results from this procedure as from a similar one applied to the rectum.

Dr. William L. Baum - I have had no experience with the apparatus of Dr. Turck, but I have been making some experiments during the last fow months at the Cook County Hospital on cases of erysipelas. In a number of cases in which there was present nephritis with scanty urine, where the patients were in a condition practically of uremic coma, with slow and labored respiration and very irregular pulse, particularly in old individuals, for want of any other therapeutic measure 1 could think of at the time, advised in one case hot injections of normal salt solution of the strength of six-tenths of 1 per cent., using a quart every two hours. This was kept up night and day for eight days, the patient receiving the injections every two hours. He passed not only large quantities of urine, but the casts diminished rapidly in number, and the patient was discharged as cured. Since that time I have had four other cases in which I have resorted to similar treatment, all of which have recovered and have been discharged as cured.
Another case in which hot water injections have been very efficacious was one that I was called to see in connection with Dr. Fütterer. It was a case of vesical hemorrhage. The hem orrhage was continuous for some forty-eight hours; there was also present hydronephrosis. The patient had at some time ulcerative endocarditis with marked symptoms of recent endocarditis. He was given hot rectal injections of the normal salt solution, as well as subcutaneous injections of one quart of normal salt solution on each side of the abdomen. In this case, as in the other cases, within almost half an hour the respiration became more regular, and the pulse also became more regular, full and strong. Whether in the latter case the marked improvement was due to the hot solution injected subcutaneously, I am not at present prepared to say. We have benefit from large quantities of hot water taken up by the mesenteric glands and the intestines, and we have rapid elimination through the kidneys. In the erysipelas cases the heat may have had a marked beneficial effect, because the change took place in an extremely short period of time.

Dr. D. W. Rogers-J was very much interested in the remarks of Drs. Kiernan and Turck with regard to surgical shock, and consider the method of Dr. Turck especially commendable. The heat supplied by the hot water which is poured into the stomach is rapidly taken up by the tissues. In addition there is a nervous stimulus, but the heat which the body must have in order to carry on its functions is supplied by this hot water, for this simple nervous stimulus would in my opinion not be sufficient to resuscitate a case suffering from severe surgical shock.

Dr. W. X. SuDDuTH-The remarks of the last speaker bring up the pointas to the causes of temperature. The old idea that temperature is entirely the result of chemic changes in the body has to be modified to a considerable extent according to recent observations. You may take an iron bar and pound it to a red heat without any chemic change taking place. It is then a matter of vibration, and so we find in the body that vibration is the principal cause of the development and main. tenance of temperature in the body, and that temperature is to a very great extent, to be controlled through mental processes. We have unquestionably much that is to be commended in the method of Dr. Turck by raising bodily temperature by the immediate contact of the hot water, which may be accounted for on the same ground, because we know vibration is much highor in hot than it is in cold water. Whether this vibration is carried and taken up by the body directly, as Dr. Turck has mentioned, in the passage of the blood through the different portions of the body and marking its course, or whether it is simply through association, raising the temperature in that way, is an open question. Undoubtedly, we have a nervous stimulation which is more effectual in the raising of the temperature and the resuscitation of the patient than we have in its mechanical effect. We have, then, the temperature raised by reason of the increased rate of nervous vibration, and the strength or the ability to maintain that vibration is the result of the increased temperature which is induced by the hot water.

The old theory of oxidization being the principal cause of temperature is pretty generally accepted, but I think we have evident reason for attributing many of the changes in temperature to mental processes. Let us take fevers, for instance, where we have a temperature of $\mathbf{1 0 4}$ degrees, by purely passify ing methods, by suggestion, the temperature can be dropped from 3 to 4 in from fifteen to twenty minutes without the use of any drug or the induction of any chemic change, that we know of, in the body. Even so winere we have a subnormal temperature, by working on the emotions, we can raise the bodily temperature a number of degrees in a few moments. While suggestion is not an important part in the treatment used by Dr. Turck, yet we can not eliminate it from any process on the living human body, even where the patient is apparently unconscious. The facts are there is no such thing as unconsciousness short of death. I have proved that, in my experiments in the administration of anesthetics, 1 was able to get a response from patients under the most profound anesthesia, simply by keeping in touch with them when they were going into the anesthetic state. So Dr. Turck's remarks and conduct with the patient must have a direct effect in the resuscitation.

Dr. James G. KiERNAN-I do not want unduly to swell the neurologic trend of the discussion at the present time, yet $I$ can not but take issue with the position that local tissue change irrespective of nerve action underlies the results, as Dr. Turck has claimed. 'This seems to be turning back the page of pathology to the days of Broussais when such a doc trine led to an abuse of venesection because fever was regarded as a result of such local change. Fever, as the researches of 
Ott of Pennsylvania, have shown, is primarily set up by an increase of heat production beyond that of heat dissipation. An agent from within or from without deranges the harmony of the thermotaxic, thermogenetic and thermolytic apparatus by which in the initial stage the metabolism of the tissue are usually increased temporarily, this increment being greater than that generated upon the restricted amount of nutriment. Once established, the fever continues not from excessive pro duction but from an altered relation between heat production and heat dissipation. Fever is not a fire kept up by an exces sive oxidation of the constituents of the economy. Thus heat production may really be subnormal, yet the bodily tempera ture may be at high fever heat. The thermotaxic centers of the brain maintain the balance between heat production and heat dissipation so as to keep the temperature at 98.4 degrees F. In fever these thermotaxic centers are disordered; it is mainly the basal thermotaxic which are so affected that dis turbance of the relation between heat production and heat dis sipation produces a higher temperature. Since the peripheral terminations of the sensory nerves influence a thermolytic center by reflex action they constitute important factors in hea regulations. It seems to me, therefore, from the description given by Dr. Turck, that the comparative rapidity with which this treatment acts would imply such a reflex action on certain vaso-motor centers and on certain heat-regulating centers, because the condition of surgical shock is essentially one of extreme heat inhibition. To reverse that condition excitation would be physiologically indicated along the line stated by Dr. Turck, He could thus excite a large number of centers quickly through the stomach. It would seem that a reverse physiologic process would be desirable, and if there be need to stimulate the thermo-excitatory centers, it can be done better by a method of this kind than by a procedure implying merely local thermic excitation, which is very slow. As to the ultimate origin of this stimulation, in all probability, like all nerve force, it is allied to vibration. But be that as it may, to refer it simply to a local chemic action would be going a long way to ccomplish a small result. Furthermore, transmission ofheat a must be more or less through the nervous system, and not direct.

Dr. William A. Prsey--In the interest of orthodox physiology and physics, I would ask Dr. Sudduth and Dr. Kiernan if they mean to maintain that the heat of the body is not the result of metabolic changes of exactly the same character as those that produce by oxidation the heat in an ordinary fire? I may have misunderstood Dr. Sudduth, but my understanding of his remarks was that the nervous system not only regulated heat, but was of great importance in the production of it; that the production of heat in the body was not, at all time, simply a question of the transformation of physical energy. Am I right?

Dr. SudDuTH - You are correct.

Dr. PusEY - The position is so contrary to the accepted doctrines of physics that it seems to me it should not be allowed to pass unnoticed. I accept all that Dr. Sudduth and Dr. Kiernan say with regard to the influence of the nervous system in regulating temperature and know that we may have all sorts of aberration of temperature when the heat center is interfered with. I accept the statement that the heat of the body is merely a form of energy - that is the point I am maintaining - but to assume that the nervous system can produce heat without a material foundation for it, can evolve de novo and from nothing material, is a position that it seems to me should not be allowed to pass unchallenged in this Academy.

Dr. Kiernan - I would ask Dr. Pusey, without attacking his position, whether he believes in such a doctrine as correlation and conservation of energy

Dr. Puser-I certainly do. It is because the positions enunciated are in conflict with this universally accepted law of the conservation of energy, that I take exception to them. I will gladly agree with the proposition that the nervous system is the thermostat, but that it is any thing more than a very significant part of the human furnace is a proposition which is so contrary to the accepted laws of physics and physiology that with the shades of Michael Foster staring me in the face I can but protest against it.

Dr. D. W. Rogers-Exactly what Dr. Pusey has said is something that came into my mind. No one will deny from the experiments that have been made during the last twenty five or fifty years, that heat is a form of vibration, and that by pounding a piece of iron we can get it red hot. As Dr. Pusey has said, the heat generated in our body comes from the food which we take into it. The idea of calling the nervous system a thermostat is an excellent one. We might consider the bod an automatic furnace, regulated and controlled by electric devices. Regarding the experiments of Dr. Turck, the hot water which he pours into the stomach supplies the place of heat made in a normal condition of the body by the chemic processes controlled by the nervous system, and this heat is not taken up by the body simply through an increased stimu lus to the nervous system, but by the transmission of vibrations from the hot water to the tissues of the body itself. Mental processes, it is true, have much to do with the produc. tion of heat, inasmuch as these processes control the nervous system, control the impulses going to various parts of the body, and if a heat inhibitory center is excited (we are supposing, for instance, we have a heat inhibitory center) the metabolic processes in various parts of the body would be checked, and the heat would be lessened.

Dr. William L. Ballanger-I have seen neurasthenic cases with cold skin, feet and hands, and I have seen them come out of this condition of shock or collapse by simply following deep breathing exercises for a few minutes. Undoubtedly others have seen the same thing. I narrate the above as suggestive. It is one of the ways in which the temperature of the body may be regulated. Dr. Turck's experiments upon animals are very instructive, showing the physical changes which take place in the blood in conditions of shock, and the changes which occur under the influence of heat applied to the stomach. His work is deserving of praise and commendation.

Dr. Turck (closing the discussion)-I have presented these experiments and clinical observations just as they appeared, and it is impossible at present to explain the phenomena completely. But there are facts here that can not be disputed. First, the congestion of the abdominal viscera in shock, and this congestion is apparently commensurate with the loss of temperature and also with the lack of tone of the nervous system. To completely analyze these phenomena would require more knowledge than we have at the present time; but the facts are stubborn, that we can not apparently experimentally produce a profound shock without the occurrence of congestion and that the reduction of such shock does not occur until we reduce the congestion and distribute blood over the surface. The blood that is being distributed over the surface is warm blood. Energy is transmitted and as this energy is carried on we see the effect upon the arterioles. I could not recite all the experiments here, because I have previously published some and others are to follow. Let us take the simple introduction of water into the colon; we often produce a condition of congestion rather than of reduction. The patient feels the shock of such a procedure. He is momentarily stimulated, but soon relaxes into a state of congestion, the hands and feet become cold, and the patient will feel weak. Whereas, if we use water of high temperature the reverse condition is produced. We have a vasomotor stimulation. Inasmuch as water at a high temperature, introduced directly into the viscera is contraindicated, we use this method of introducing it through a tube, and if $I$ may use another term, the absorption of that heat is constant. I have presented these facts as possibly opening up further investigation in determining the exact cause of the phenomena of shock and also the rationale of the reduction of shock.

\section{INTESTINAL ANASTOMOSIS}

Read before the Illinois State Medical society, May, 1897. BY JACOB FRANK, M.D.

SURGEON TO THE ST. ELIZABETH AND GERMAN HOSPYTALS.

$$
\text { CHICAGO, ILL. }
$$

The subject which I am about to touch upon is an ever interesting one. I would like to relate the early history of our instructors in surgery, in the line of intestinal anastomosis, but were I to attempt such a task I would be called a monopolist of time. Suffice it for me to state, that they, even 150 years ago, realized the necessity of some speedy and safe method for dealing with this most important branch of surgery. They were evidently not satisfied with the suture method, as is manifest by their numerous attempts to call unto their aid mechanical means, such as steel rings, rawhide, turnips, bone, catgut, rings, gelatin, etc. Denan, in an article in "Receuil de la Société Royale de Médecine de Marseille," l'an 1826, described his device for intestinal anastomosis, consisting of steel rings connected with a spring, and laid stress upon the pressure necrosis to be obtained from 